\title{
Implications of Frailty for Peritransplant Outcomes in Kidney Transplant Recipients
}

\author{
Xingxing S. Cheng ${ }^{1} \cdot$ Krista L. Lentine ${ }^{2,3} \cdot$ Farrukh M. Koraishy ${ }^{2} \cdot$ Jonathan Myers $^{4} \cdot$ Jane C. Tan $^{1}$
}

Published online: 26 January 2019

(C) The Author(s) 2019

\begin{abstract}
Purpose of Review Research over the past few decades points to the importance of frailty, or the lack of physiologic reserve, in the natural history of chronic diseases and in modifying the impact of potential interventions. End-stage kidney disease (ESKD) and the intervention of kidney transplantation are no exception. We review the recent epidemiologic and cohort-based evidence on the association between frailty and kidney transplant outcomes and provide a framework of questions with which to approach future research endeavors and clinical practice.

Recent Findings Frailty in kidney transplant candidates can be measured in numerous ways, including descriptive phenotype, description scores, functional testing, and surrogate measures. Regardless of the metric, the presence of frailty is strongly associated with inferior pre- and posttransplant outcomes compared with the absence of frailty. However, some frail patients with ESKD can benefit from transplant over chronic dialysis. Evidence-based approaches for identifying frail ESKD patients who can benefit from transplant over dialysis, with acceptable posttransplant outcomes, are lacking. Interventional trials to improve frailty and physical function before transplant (prehabilitation) and after transplant (rehabilitation) are also lacking.

Summary Frailty is increasingly recognized as highly relevant to peritransplant outcomes, but more work is needed to (1) tailor management to the unique needs of frail patients, both pre- and posttransplant; (2) define phenotypes of frail patients who are expected to benefit from transplant over dialysis; and (3) develop interventions to reverse frailty, both pre- and posttransplant.
\end{abstract}

Keywords Assessment $\cdot$ Frailty $\cdot$ Kidney transplant $\cdot$ Outcomes $\cdot$ Prehabilitation $\cdot$ Survival

\section{Introduction}

Frailty is a state of decreased physiologic reserve and diminished ability to recover from physiologic stressors [1]. Functional status may decline and fail to recover after a medical illness or intervention, placing the individual at higher risk

This article is part of the Topical Collection on Frailty and Gerontology

Krista L. Lentine

krista.lentine@health.slu.edu

1 Department of Medicine, Division of Nephrology, Stanford University, Stanford, CA, USA

2 Department of Medicine, Division of Nephrology, Saint Louis University, St. Louis, MO, USA

3 Center for Abdominal Transplantation, Saint Louis University, 1402 S. Grand Blvd., St. Louis, MO 63104, USA

4 Department of Medicine, Division of Cardiology, Palo Alto VA Hospital and Stanford University, Palo Alto, CA, USA for complications. Literature on frailty and kidney transplant outcomes uses a wide range of definitions and myriad of metrics including the following:

- descriptive phenotype (Fried criteria [2]);

- descriptive scores (Frailty Index [3], health-related quality of life [HRQOL] scores [4]);

- physical function testing (SPPB [5], measures of lower extremity strength and grip strength [6]);

- cardiopulmonary exercise testing (peak $\mathrm{VO}_{2}$ ) [7], other functional tests (6-minute walk test, sit-to-stand, or timed walking tests [8]);

- other surrogate measures (days of hospitalization [9•], falls [10]).

Frailty exists on a spectrum, ranging from mild decrements in reserve (sometimes termed "prefrailty") to severe functional impairment [1]. Frailty has been associated with poor health outcomes in almost all conditions, from community-dwelling older adults [1] to solid organ transplant recipients [11]. 
Frailty is common in kidney transplant candidates. Studies in dialysis-dependent patients suggest a frailty prevalence of up to 70\% [12-14]. Even among kidney transplant candidates, generally the healthiest of dialysis-dependent patients, approximately $20 \%$ meet criteria for frailty [11]. Advanced chronic kidney disease (CKD)/uremia and commonly associated comorbid conditions, including anemia, diabetes mellitus, and heart disease, all contribute to the frailty phenotype [15]. Understanding frailty and its effect on transplant outcomes therefore has significant implications for patient education and clinical management, including the pursuit of and acceptance for kidney transplantation as a treatment option.

To better assess and utilize frailty in the peritransplant clinical setting, the following questions need answers:

1. Is there a frailty threshold at which the risk of transplantation exceeds that of continuing maintenance dialysis?

2. Should the type of transplant considered be tailored based on frailty status? For example, should some frail candidates only consider living donor kidney transplantation to minimize the risks associated with delayed graft function and further deterioration in the posttransplant course?

3. To what extent is frailty reversible after transplant, and how long does this process take?

4. What interventions may be effective to mitigate frailty, both pre- and posttransplant?

This review seeks to illuminate some of the above issues. Herein, we will review the associations between frailty and transplant outcomes, an active research area over the last 510 years. We then seek to apply that knowledge to the questions of pre- and posttransplant management of frail patients and highlight knowledge deficits to be addressed by future research efforts.

\section{Frailty and Outcomes Before Transplant}

Emerging literature on the association between frailty and outcomes in kidney transplant candidates and recipients is predominantly observational. These studies demonstrate a higher risk of mortality and morbidity among patients who are frail compared with non-frail patients, both before and after transplantation. It is important to note, however, that under the current candidate selection practices, survival benefit with transplantation may be seen even among some candidates who are frail.

Multiple studies have established an association between patient-reported or surrogate measures of frailty and adverse outcomes in transplant candidates on the waiting list. In a large multi-center cohort study of 1975 patients, the Fried frailty phenotype is associated with higher waitlist mortality (HR $1.26^{2.19} .79$ ) [16]. In a large retrospective study using registry data, Reese et al. [17] noted that kidney transplant candidates in the lowest baseline physical function score quartile (based on the Medical Outcomes Study 36-Item Short Form Health Survey-SF-36) were less likely to undergo transplantation, more likely to be inactivated and have a lower survival at 3 years compared with the highest quartile ( $84 \%$ vs $92 \%$ ). Survival benefit conferred by transplantation persisted in every physical function quartile. Limitations of the study include selection bias of patients both for waitlisting and for transplantation, and the use of indirect measures of physical functioning, albeit ones that have been validated in the dialysisdependent population. In another registry data-based study, Lynch et al. [9•] studied whether hospital days in the first year of waitlist can be used as a measure of fitness for transplant. Based on registry data for 51,111 patients, those with higher hospitalization burden were noted to have higher waitlist mortality (1-7 hospital days, HR $1.201 .24_{1.28} ; 8-14$ days, HR $1.421 .49_{1.56} ; \geq 15$ days, HR ${ }_{1.99} 2.07_{2.15}$; versus 0 days). Those with a high hospitalization burden had lower posttransplant survival, but survival was significantly better than remaining on the waitlist. Furthermore, in a singlecenter study of 96 transplant candidates, Locke et al. [18] observed that lean muscle mass (measured via morphometric assessments of psoas muscle attenuation and paraspinous lean volume) was associated with a small but significant decreased risk of death ( $\mathrm{HR}_{0.91} 0.93_{0.96}$ for higher psoas muscle attenuation and $\mathrm{HR}_{0.96} 0.98_{0.99}$ for increase in lean paraspinous volume) over a 5-6-year follow-up period. Together, these studies demonstrate that the association between frailty and pretransplant mortality is robust across different frailty measures. Frailty assessments may be particularly important where the patient comorbidity burden is lower, in identifying high-risk patients who may not be noticed otherwise [19]. These data also show that some frail ESKD patients can benefit from transplant over chronic dialysis, although how to identify those who will benefit and have acceptance posttransplant outcomes is not yet known.

One study applied an objective measure of cardiovascular reserve and arrived at a similar conclusion. Ting et al. [20] studied 240 waitlisted patients followed for 5 years. They quantified cardiovascular reserve using cardiopulmonary exercise testing and found that patients with an impaired peak $\mathrm{VO}_{2}$ (based on percentage of age-predicted peak $\mathrm{VO}_{2}$ ) had a significantly lower survival compared with those with a better reserve. However, among patients with low cardiovascular reserve, transplanted patients had significantly greater survival compared with non-transplanted patients $\left(\mathrm{HR}={ }_{0.09} 0.22_{0.56}\right)$.

\section{Frailty and Outcomes After Transplant}

Studies of posttransplant outcomes in transplant recipients have utilized both the Fried frailty phenotype and other 
measures of frailty. Outcomes examined include short-term (delayed graft function and hospitalization) and long-term (death) measures (Table 1). Frailty, as assessed by varying metrics in these studies, is associated with worse short-term outcomes after transplant. Frailty defined by the Fried index, a combination of self-reported and objective measures, was associated with an almost twofold increase in risk of delayed graft function, 1.2-fold increase in risk of protracted initial hospital stay, and 1.6-fold increase in risk of hospital readmission within 1 month [28]. The effect of frailty on length of stay is especially pronounced in patients with depressive symptoms (1.9-fold increase in risk) [29]. Pretransplant hospitalization, a surrogate measure of frailty, is also associated with increased posttransplant hospitalization ( 0 hospital days, $73 \%$; 1-7 days, $70 \%$; $8-14$ days, $75 \%$; $\geq 15$ days, $80 \%$ hospitalization by 12 months of transplant) [30].

Frailty is also associated with long-term transplant outcomes, including mortality and graft survival. The association is strong, whether frailty is measured by the Fried index [11] or other metrics. Among the five components of the Fried index, the combination of poor grip strength, low physical activity, and slow walking speed was especially strongly associated with increased mortality $\left(\mathrm{HR}_{1.14} 2.61_{5.97}\right)$ [24]. Other studies employed alternative measures of frailty, including the 6-minute walk test [27], Short Physical Performance Battery [26], morphometric age [31], and hospitalization in the first year of waitlist [30]. All these studies confirmed the strong association with frailty measures and posttransplant mortality (compared with the absence of frailty). Importantly, the studies available to date are observational, and how frailty should inform transplant candidacy is currently controversial.

\section{Frailty and Transplant Candidacy}

The kidney implantation procedure and peritransplant immunosuppression represent significant physiologic stressors, from which the frail recipient may, by definition, have a protracted recovery (Fig. 1). During the recovery period, complications may arise which further reduce physical performance. McAdams-DeMarco et al. [32] outlined mechanisms by which frail transplant recipients may be prone to transplant complications, including increased hospitalization, immunosuppression intolerance [33], and delirium [34]. A threshold, or thresholds, for frailty may exist for which certain frail candidates are better off remaining on dialysis and others should only accept living donor kidneys to minimize the extent of peritransplant physiologic stress. Indeed, approximately $5 \%$ of transplant candidates were removed from the waitlist in 2016 because they were too sick for transplant [35]; many of them may have an unacceptably high level of irreversible frailty. The only existing surrogate measure for frailty/physical function that is mandated reporting nationally is the Karnofsky Scale, which is a rough surrogate [36]. Lack of a systematic approach and standardized instruments to assess frailty in transplant candidates makes it difficult to determine and evaluate frailty thresholds for informing transplant candidacy.

Preliminary data suggest that, under current practices at certain centers, measures of frailty may improve posttransplant. In a cohort of 349 transplant recipients at Johns Hopkins Hospital [23•], 20\% had the frailty phenotype (meeting at least 3 of the Fried criteria), and a higher percentage became frail within 12 months of transplant. At 3-month posttransplant, $74 \%$ of the initially frail recipients became less frail. The choice of the Fried phenotype, a non-quantitative instrument, to quantify frailty improvement is a limitation of this study, as is the high likelihood of selection bias in the study protocol. Longitudinal fluctuations in measuring the frailty phenotype may also account for part of the observed improvement. In a follow-up study of 443 transplant recipients from Johns Hopkins and University of Michigan Hospital [25], the physical and kidney disease-specific health-related quality of life (HRQOL) scores posttransplant improved in both initially frail and non-frail recipients. The improvement was more marked in frail recipients, especially in the domains of general health, effects of kidney disease, cognitive function, and social interaction. At these study centers, most frail transplant candidates appear to recover posttransplant and experience improvements in functional status and quality of life. Relatedly, in a large registry study, even patients with low physical function scores experience a survival advantage conferred by transplant over dialysis [17], but this finding may be affected by unmeasured selection factors. Replication of these studies using quantitative frailty instruments, pre-specified assessment intervals, and longer followup at different transplant centers (with different thresholds for recipient and organ acceptance and different transplant protocols) will confirm (or disprove) these initial observations and lend empirical credence to our proposed paradigm in Fig. 1.

If most frail transplant recipients improve posttransplant, then the higher mortality and adverse outcome rates in frail recipients may be attributed to either stochastic posttransplant events or the presence of patient subsets whose posttransplant trajectories diverge from non-frail patients. Predicting these "high-risk" frail candidates may enable us to refine transplant candidacy criteria and avoid the unfortunate outcome of making a patient worse with a failed transplant. All existing studies on this topic confront the inescapable limitations that (1) stochastic posttransplant events cannot be predicted; (2) a strong selection bias exists, as perceived frailty is already contributing to decisions of transplantation; and (3) a model to be used in shared decision making regarding whether to proceed with a transplant or remain on maintenance dialysis will need an extremely high degree of accuracy (i.e., ability to provide the correct estimates for the probability of an adverse outcome). Existing models include the Estimated Post-Transplant 


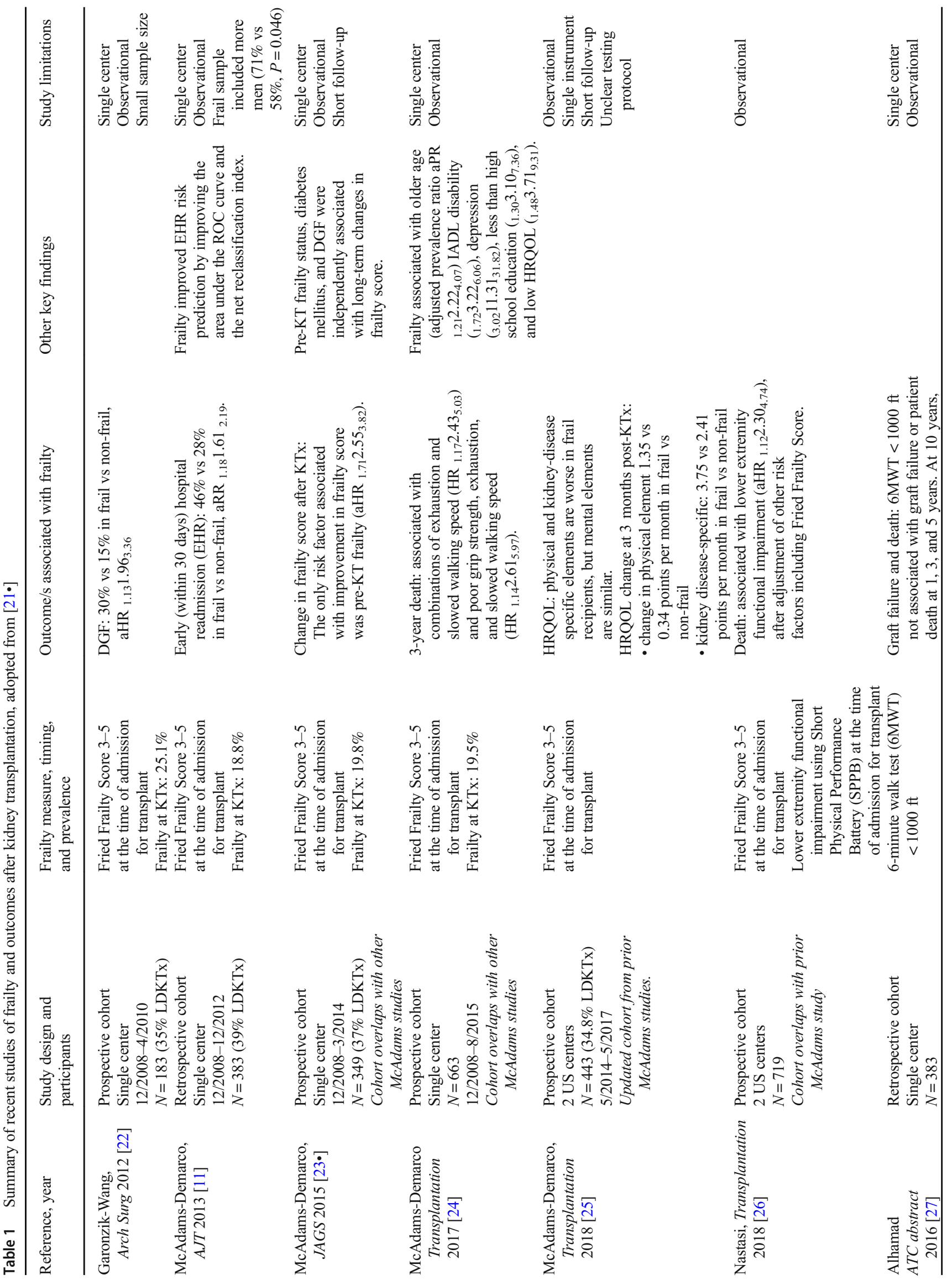




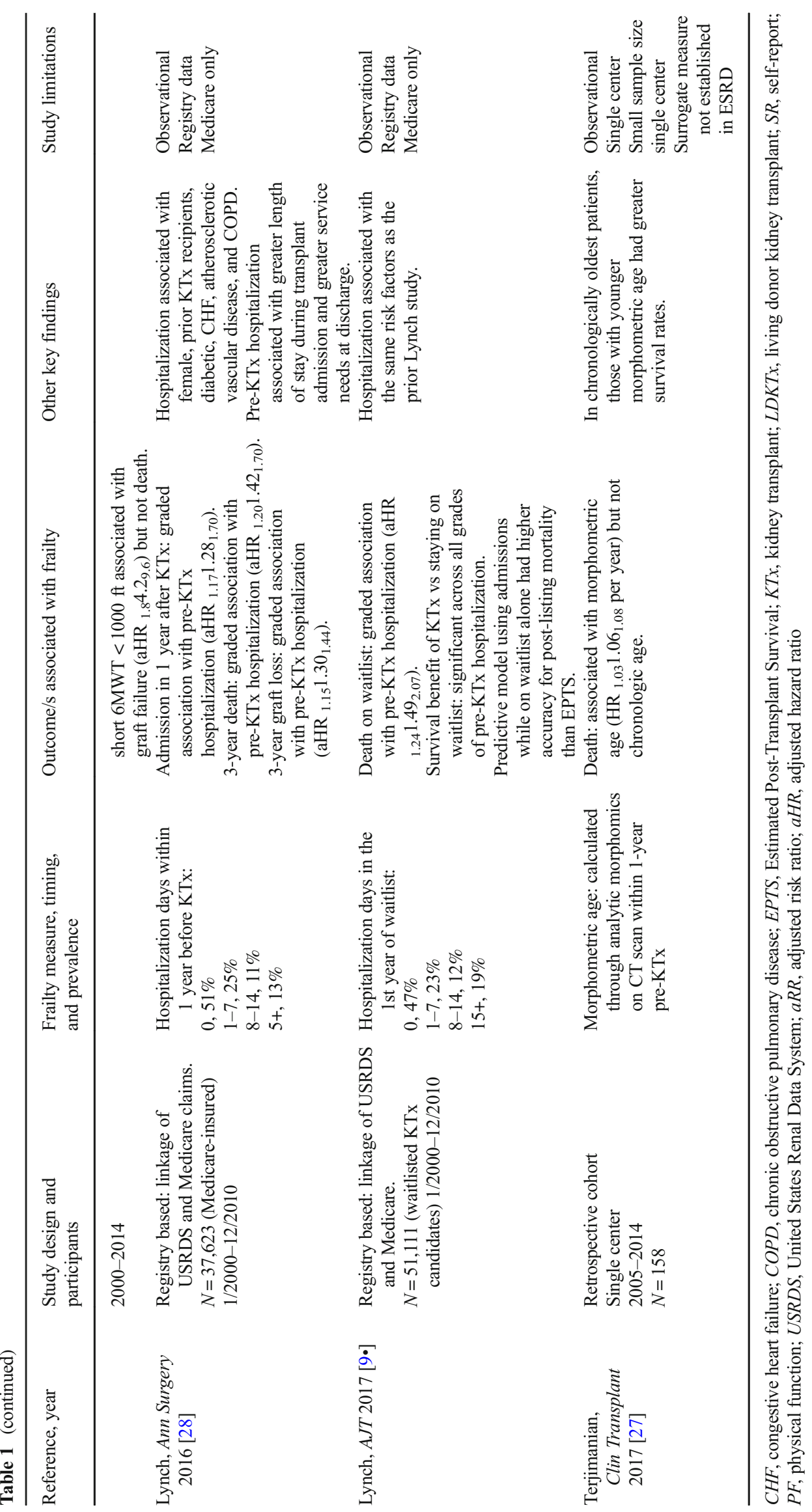




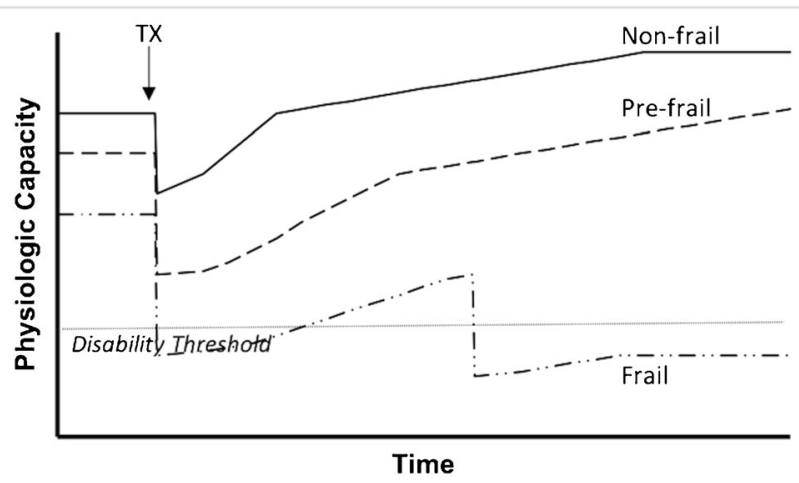

Fig. 1 A schematic of frailty as the loss of functional reserve. A non-frail patient (solid line) receives a kidney transplant (arrow): physiologic capacity decreases postoperatively and recovers to a better baseline than pretransplant. A prefrail patient (hashed line) experiences a greater decline and slower recovery of physiologic capacity, but ultimately achieves better physiologic capacity than pretransplant. A frail patient (dotted and hashed line) experiences a great decline in physiologic capacity to the point of losing dependence (horizontal dotted line), at which point recovery is prolonged and also plagued by further setbacks, which eventually results in a permanent loss of functional independence and poor outcome. TX, transplant

Survival (EPTS) score [37] which accounts for only age and limited comorbidities (diabetes, length of time on dialysis, and prior transplant), and various models including measures of frailty $[9 \bullet, 11,36]$. In the most discriminating of these models [9•] (based on days of hospitalizations in the first year of waitlist), recipients in the highest category of risk have 3year death or graft loss rates of 30\%: whether such a failure rate warrants proceeding with kidney transplant is a decision to be made at the individual level by shared decision making.

\section{Timing of Frailty Assessments}

The timing of pretransplant frailty assessment warrants brief discussion. Literature to date has reported frailty measures at one pretransplant time point, mostly immediately prior to transplant [11] or at study enrollment [20]. Such timing is practical and useful for research studies but requires modification for useful clinical practice. Measuring frailty at the time of transplant evaluation can help inform transplant candidacy and type of transplant offered but may miss deceased donor transplant candidates who become frail while awaiting transplant [38]. Measuring frailty immediately prior to transplant can help guide posttransplant management but will have little bearing on decisions and counseling regarding transplant candidacy. Ideally, frailty will be assessed longitudinally while awaiting a transplant and more frequently as candidates move toward the top of the waitlist (Fig. 2). Such reassessment should be framed in the context of other patient factors, such as chronologic age,

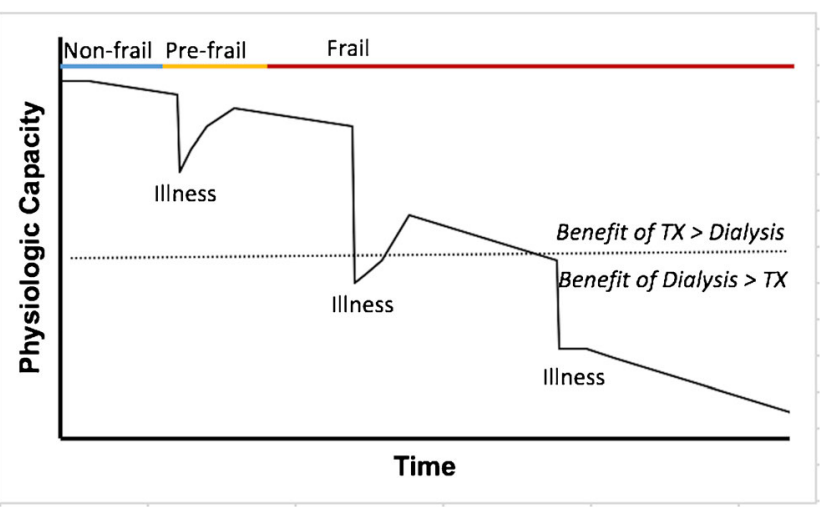

Fig. 2 A schematic of the functional trajectory of a patient on the kidney transplant waitlist. Re-assessments, especially after major illnesses, are crucial in properly phenotyping patients on the frailty spectrum and making the appropriate transplant-related decisions. TX, transplant; Benefit, projected benefit of transplant or dialysis

social support, and other comorbidities. However, for the vast majority of transplant candidates whose primary or nephrology care is not delivered by transplant center affiliates, repeat frailty testing will necessitate either repeat visits to transplant centers or close coordination between transplant centers and local nephrology practices and dialysis units. Implementation of full, longitudinal pretransplant frailty monitoring will require a more integrated care coordination model than what is currently available.

\section{Frailty and Pretransplant Management}

In addition to better assessment of frail patients with ESKD who are expected to benefit from transplant, effective interventions to modify frailty may allow more frail patients to become suitable transplant candidates. However, the optimal strategy to address frailty before transplantation is not known. Physical activity interventions, with or without nutritional interventions, have demonstrated reduction of frailty measures in communitydwelling elderly adults [39]. Whether this can be consistently achieved in the advanced CKD population is debatable. Physical or exercise therapy delivery in the advanced CKD population is challenging. Implementation of intradialytic or supervised interdialytic exercise is limited by staffing challenges, competing priorities, and reimbursement [40, 41]. Home-based exercise therapy may result in modest improvements in 6-minute walk test performance and in lower extremity strength [42•] How the positive effects persist beyond the study period is unknown. The myriad of barriers to exercise reported by patients, including fatigue, comorbidities, and limitations related to dialysis access [43], may explain the high attrition rate $(20-50 \%)$ reported in most exercise intervention studies in the CKD population (cf Table 1 in review by Cheng et al. [21•]. Prehabilitation, or physical rehabilitation completed 


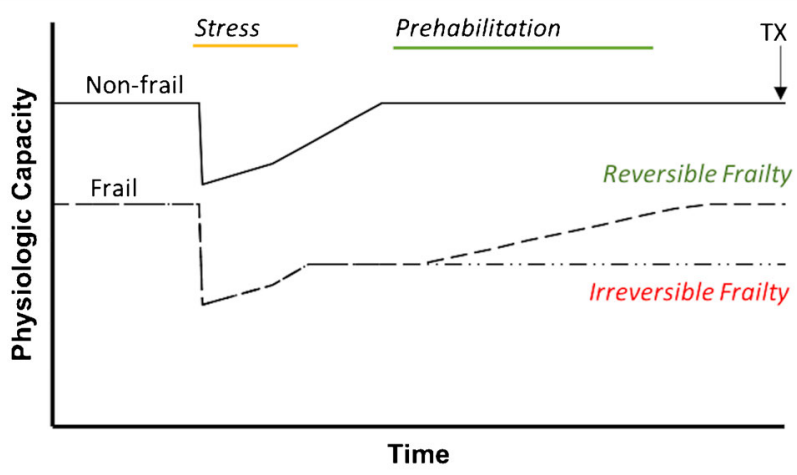

Fig. 3 A schematic of the functional trajectories of non-frail, reversibly frail, and irreversibly frail patients on the kidney transplant waitlist. A trial of prehabilitation may be useful in distinguishing the reversibly frail from irreversibly frail patient before transplant occurs

prior a major procedural intervention, has shown some success in intra-abdominal surgeries (cf Table 2 in review by Cheng et al. [21•]). In the kidney transplant setting, prehabilitation may theoretically leverage the higher motivation of patients at the top of the waitlist, but this conjecture has yet to be empirically evaluated. Response to prehabilitation, or lack thereof, may also provide an additional data point for assessing a frail patient's ongoing kidney candidacy (Fig. 3). For all these reasons, prehabilitation in kidney transplantation warrants more study.

\section{Frailty and Posttransplant Management}

There are limited data to inform modification of posttransplant care based on frailty status. Potential modifications include the following:

- Aggressive and planned physical rehabilitation after transplantation in high-risk candidates

- Immunosuppression modification

- Accommodation of patients with cognitive deficits

Compared with prehabilitation, posttransplant rehabilitation is potentially easier to arrange logistically. Two randomized controlled exercise trials exist. In a US trial [44] $(N=54$ in exercise arm), an individualized home exercise regimen, tele-monitored via phone, improved objective and selfreported physical functioning over usual care. The average age of transplant patients was quite low ( $40 \pm 13$ in the exercise arm), and no frailty screening was done at recruitment. A small UK pilot trial [45] ( $N=13$ in exercise arm) recruited older patients within 1 year of transplant and tested the effects of 12 weeks of supervised structured exercise classes twice per week. They reported a statistically non-significant trend toward an improvement in peak $\mathrm{VO}_{2}$ attributable to aerobic training that persisted for 6 months beyond the intervention end date. Replication of these studies with higher numbers
Fig. 4 Frailty considerations for key stakeholders

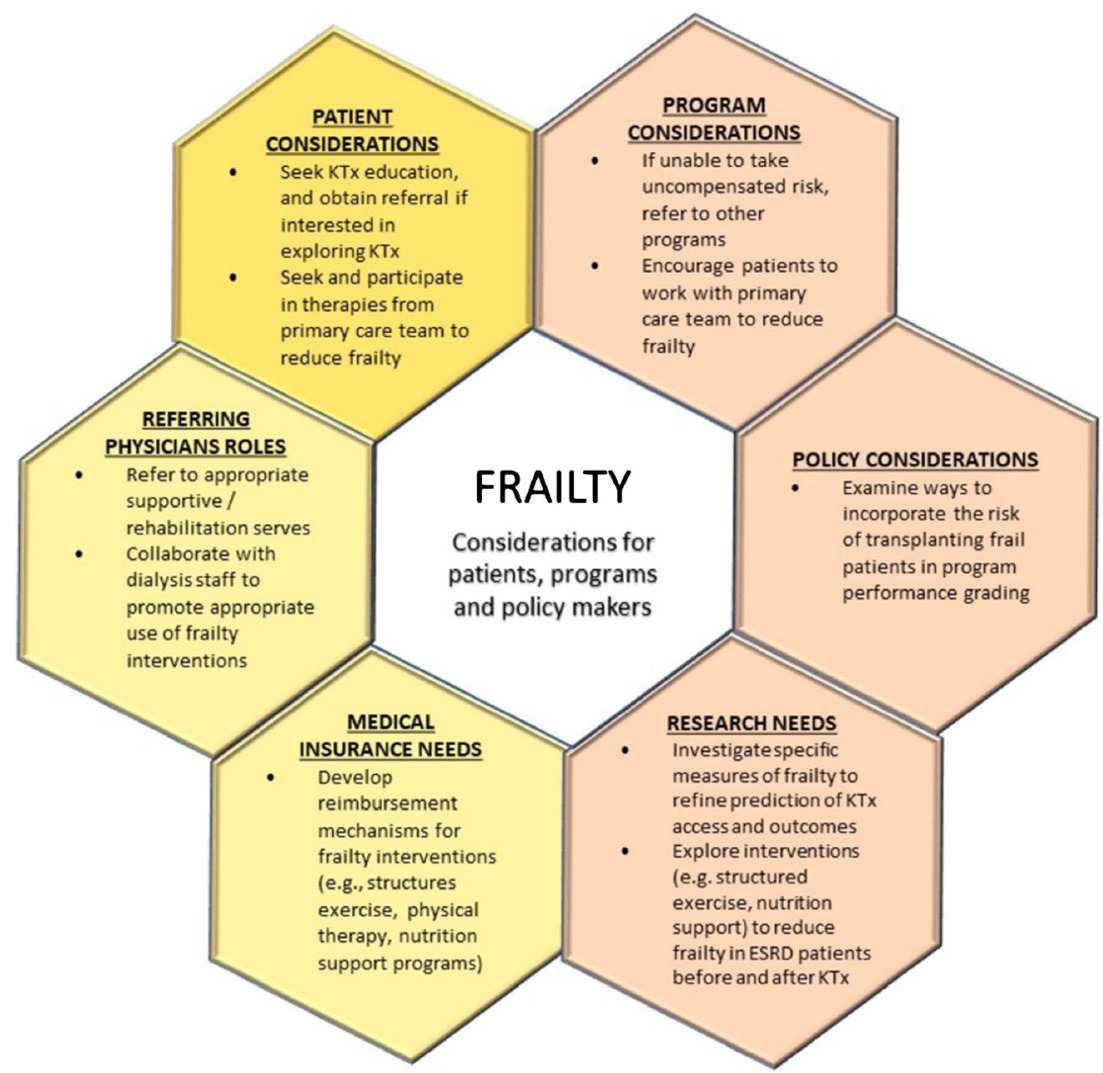


stratified by pretransplant frailty will help to delineate the benefits of rehabilitation and is necessary for obtaining insurer approval for covering the service.

The optimal approach to immunosuppression may differ between elderly frail and non-frail, non-elderly kidney transplant recipients [46*]. The altered pharmacokinetics of medications in elderly individuals may alter their exposure to immunosuppressants. Rejection and death-censored graft failure rates decrease with increasing recipient age [47, 48], implying age-related immune senescence or heightened immunosuppression exposure in elderly patients under current protocols. Side effects and immunosuppressant intolerance are also more common in frail individuals [33]. Overall, the balance between suppressing alloimmunity and minimizing side effects may call for lower immunosuppression in frail transplant recipients, but this hypothesis warrants focused studies.

Transplant centers under-recognize cognitive deficits, such as delirium, to which frail transplant recipients are particularly prone [34]. Targeted efforts to address cognitive deficits in frail transplant recipients include better delirium prevention and treatment along with targeted measures for medication safety and adherence. These are therefore reasonable steps to mitigate the downstream effects of frailty, although the optimal approach remains unknown.

\section{Conclusions}

As has been recognized in other populations, frailty is an important determinant of outcomes in kidney transplant candidates and recipients and exerts a significant impact on a patient's course, both pre- and posttransplant. In the past 510 years, a proliferation of studies has demonstrated a robust link between frailty, measured by varying metrics, and transplant outcomes. These studies also provide insight into the mechanistic basis for the link and suggest possible intervention venues, including prehabilitation, rehabilitation, immunosuppression modification, and closer attention to cognitive impairment. The observation that frailty may not preclude benefit from transplant over dialysis argues for liberal referral to transplant centers, allowing programs to make candidacy determinations, rather than denying referral based on perception that a patient may be too unfit. At this time, minimization of pretransplant dialysis exposure through early referral, effective education on the potential benefits of living donor transplantation, and consideration of non-standard deceased donor organs to increase transplant options are particularly important to elderly and frail patients. Defining characteristics of frail patients who can benefit from transplant over dialysis with acceptable posttransplant outcomes is a vital research priority. Prospective studies of interventions and management strategies to improve frailty and mitigate adverse outcomes are also needed. All stakeholders-including patients, referring physicians, transplant programs, policy makers, insurers, and researchers - should recognize the importance of frailty as a determinant of kidney transplant success and convene on interventions to improve transplant outcomes (Fig. 4).

Acknowledgments XSC, KLL, and JCT are members of the Frailty Consensus Workgroup of the American Society of Transplantation (AST) Kidney-Pancreas Community of Practice (KPCOP); KLL is the American Society of Nephrology (ASN) Quality Committee representative to the Workgroup.

Funding Information JCT funding by John M. Sobrato Foundation.

\section{Compliance with Ethical Standards}

Conflict of Interest The authors report no conflicts of interest as defined by ICMJE.

Human and Animal Rights and Informed Consent This article does not contain any studies with human or animal subjects performed by any of the authors.

Abbreviations CKD, Chronic kidney disease; EPTS, Estimated PostTransplant Survival; ESKD, End-stage kidney disease; HRQOL, Health-related quality of life

Open Access This article is distributed under the terms of the Creative Commons Attribution 4.0 International License (http:// creativecommons.org/licenses/by/4.0/), which permits unrestricted use, distribution, and reproduction in any medium, provided you give appropriate credit to the original author(s) and the source, provide a link to the Creative Commons license, and indicate if changes were made.

Publisher's Note Springer Nature remains neutral with regard to jurisdictional claims in published maps and institutional affiliations.

\section{References}

Papers of particular interest, published recently, have been highlighted as:

- Of importance

1. Clegg A, Young J, Iliffe S, Rikkert MO, Rockwood K. Frailty in elderly people. Lancet. 2013;381(9868):752-62. https://doi.org/10. 1016/S0140-6736(12)62167-9.

2. Fried LP, Tangen CM, Walston J, et al. Frailty in older adults: evidence for a phenotype. J Gerontol. 2001;56(3):M146-56.

3. Mitnitski AB, Graham JE, Mogilner AJ, Rockwood K. Frailty, fitness and late-life mortality in relation to chronological and biological age. BMC Geriatr. 2002;2:1.

4. Johansen KL, Dalrymple LS, Glidden D, Delgado C, Kaysen GA, Grimes B, et al. Association of performance-based and self-reported function-based definitions of frailty with mortality among patients receiving hemodialysis. Clin J Am Soc Nephrol. 2016;11(4):626-32.

5. Guralnik JM, Simonsick EM, Ferrucci L, Glynn RJ, Berkman LF, Blazer DG, et al. A short physical performance battery assessing lower extremity function: association with self-reported disability and prediction of mortality and nursing home admission. $\mathrm{J}$ Gerontol. 1994;49(2):M85-94. 
6. Stevens PJ, Syddall HE, Patel HP, Martin HJ, Cooper C, Aihie SA. Is grip strength a good marker of physical performance among community-dwelling older people? J Nutr Health Aging. 2012;16(9):769-74.

7. Ross R, Blair SN, Arena R, Church TS, Després JP, Franklin BA, et al. Importance of assessing cardiorespiratory fitness in clinical practice: a case for fitness as a clinical vital sign: a scientific statement from the American Heart Association. Circulation. 2016;134(24):e653-99.

8. Guyatt GH, Sullivan MJ, Thompson PJ, Fallen EL, Pugsley SO, Taylor DW, et al. The 6-minute walk: a new measure of exercise capacity in patients with chronic heart failure. Can Med Assoc J. 1985;132(8):919-23.

9. Lynch RJ, Zhang R, Patzer RE, Larsen CP, Adams AB. First-year waitlist hospitalization and subsequent waitlist and transplant outcome. Am J Transplant. 2017;17(4):1031-41. A powerful and comprehensive registry analysis illustrating how first-year waitlist hospitalization may be used as a surrogate for adverse waitlist and transplant outcomes.

10. Plantinga LC, Lynch RJ, Patzer RE, Pastan SO, Bowling CB. Association of serious fall injuries among United States end stage kidney disease patients with access to kidney transplantation. Clin J Am Soc Nephrol. 2018;13(4):628-37.

11. McAdams-DeMarco MA, Law A, King E, et al. Frailty and mortality in kidney transplant recipients. Am J Transplant. 2015;15(1): 149-54.

12. Bao Y, Dalrymple L, Chertow GM, Kaysen GA, Johansen KL. Frailty, dialysis initiation, and mortality in end-stage renal disease. Arch Intern Med. 2012;172(14):1071-7.

13. Johansen KL, Chertow GM, Jin C, Kutner NG. Significance of frailty among dialysis patients. J Am Soc Nephrol JASN. 2007;18(11):2960-7.

14. McAdams-DeMarco MA, Law A, Salter ML, et al. Frailty as a novel predictor of mortality and hospitalization in individuals of all ages undergoing hemodialysis. J Am Geriatr Soc. 2013;61(6): 896-901.

15. Kim JC, Kalantar-Zadeh K, Kopple JD. Frailty and protein-energy wasting in elderly patients with end stage kidney disease. J Am Soc Nephrol. 2013;24(3):337-51.

16. McAdams-DeMarco MA, Ying H, Thomas AG, et al. Frailty, inflammatory markers, and waitlist mortality among patients with end-stage renal disease in a prospective cohort study. Transplantation. 2018;102(10):1740-6.

17. Reese PP, Shults J, Bloom RD, Mussell A, Harhay MN, Abt P, et al. Functional status, time to transplantation, and survival benefit of kidney transplantation among wait-listed candidates. Am J Kidney Dis. 2015;66(5):837-45.

18. Locke JE, Carr JJ, Nair S, et al. Abdominal lean muscle is associated with lower mortality among kidney waitlist candidates. Clin Transplant. 2017;31(3). https://doi.org/10.1111/ctr.12911.

19. Parez Fernandez M, Martinez Miguel P, Ying H, et al. Comorbidity, frailty, and waitlist mortality among kidney transplant candidates of all ages. Am J Nephrol. 2019;49(2):103-110.

20. Ting SMS, Iqbal H, Kanji H, Hamborg T, Aldridge N, Krishnan N, et al. Functional cardiovascular reserve predicts survival pre-kidney and post-kidney transplantation. J Am Soc Nephrol. 2014;25(1): 187-95.

21. Cheng XS, Myers JN, Chertow GM, et al. Prehabilitation for kidney transplant candidates: is it time? Clin Transplant. 2017;31(8). Review article with summary tables for evidence related to physical therapy in patients on dialysis and related to prehabilitation in abdominal surgery.

22. Garonzik-Wang JM, Govindan P, Grinnan JW, Liu M, Ali HM, Chakraborty A, et al. Frailty and delayed graft function in kidney transplant recipients. Arch Surg. 2012;147(2):190-3.
23. McAdams-DeMarco MA, Isaacs K, Darko L, et al. Changes in frailty after kidney transplantation. J Am Geriatr Soc. 2015;63(10):2152-7. The first study to report longitudinal changes in frailty markers after kidney transplant.

24. McAdams-DeMarco MA, Ying H, Olorundare I, et al. Individual frailty components and mortality in kidney transplant recipients. Transplantation. 2017;101(9):2126-32.

25. McAdams-DeMarco MA, Olorundare IO, Ying H, et al. Frailty and postkidney transplant health-related quality of life. Transplantation. 2018;102(2):291-9.

26. Nastasi AJ, McAdams-DeMarco MA, Schrack J, et al. Pre-kidney transplant lower extremity impairment and post-kidney transplant mortality. Am J Transplant. 2018;18(1):189-96.

27. Alhamad T, Lentine K, Anwar S, et al. Functional capacity pretransplantation measured by 6 minute walk test and clinical outcomes. ATC Abstracts https://atcmeetingabstracts.com/abstract/ functional-capacity-pre-transplantation-measured-by-6-minutewalk-test-and-clinical-outcomes/. Accessed August 1, 2018.

28. McAdams-DeMarco MA, Law A, Salter ML, et al. Frailty and early hospital readmission after kidney transplantation. Am J Transplant. 2013;13(8):2091-5.

29. Konel JM, Warsame F, Ying H, Haugen CE, Mountford A, Chu $\mathrm{NM}$, et al. Depressive symptoms, frailty, and adverse outcomes among kidney transplant recipients. Clin Transpl. 2018;32(10): e13391.

30. Lynch RJ, Zhang R, Patzer RE, Larsen CP, Adams AB. Waitlist hospital admissions predict resource utilization and survival after renal transplantation. Ann Surg. 2016;264(6):1168-73.

31. Terjimanian MN, Underwood PW, Cron DC, et al. Morphometric age and survival following kidney transplantation. Clin Transplant. 2017;31(10).

32. McAdams-DeMarco MA, King EA, Luo X, et al. Frailty, length of stay, and mortality in kidney transplant recipients: a national registry and prospective cohort study. Ann Surg. 2017;266(6):1084-90.

33. McAdams-DeMarco MA, Law A, Tan J, et al. Frailty, mycophenolate reduction, and graft loss in kidney transplant recipients. Transplantation. 2015;99(4):805-10.

34. Haugen CE, Mountford A, Warsame F, Berkowitz R, Bae S, G. Thomas A, et al. Incidence, risk factors, and sequelae of postkidney transplant delirium. J Am Soc Nephrol. 2018;29(6):1752-9.

35. Hart A, Smith JM, Skeans MA, OPTN/SRTR, et al. Annual data report: kidney. Am J Transplant. 2016;18(S1):18-113.

36. Bui K, Kilambi V, Rodrigue JR, Mehrotra S. Patient functional status at transplant and its impact on posttransplant survival of adult deceased-donor kidney recipients. Transplantation. 2018.

37. Clayton PA, McDonald SP, Snyder JJ, Salkowski N, Chadban SJ. External validation of the estimated posttransplant survival score for allocation of deceased donor kidneys in the United States. Am J Transplant. 2014;14(8):1922-6.

38. Chu NM, Deng A, Haugen CE, Segev DL, McAdams-DeMarco MA. Dynamic frailty before kidney transplantation - time of measurement matters. Transplantation In press.

39. Puts MTE, Toubasi S, Andrew MK, Ashe MC, Ploeg J, Atkinson E, et al. Interventions to prevent or reduce the level of frailty in community-dwelling older adults: a scoping review of the literature and international policies. Age Ageing. 2017;46(3):383-92. https:// doi.org/10.1093/ageing/afw247.

40. Kontos PC, Miller K-L, Brooks D, Jassal SV, Spanjevic L, Devins GM, et al. Factors influencing exercise participation by older adults requiring chronic hemodialysis: a qualitative study. Int Urol Nephrol. 2007;39(4):1303-11.

41. Thompson S, Tonelli M, Klarenbach S, Molzahn A. A qualitative study to explore patient and staff perceptions of intradialytic exercise. Clin J Am Soc Nephrol. 2016;11(6):1024-33.

42. Manfredini F, Mallamaci F, D'Arrigo G, et al. Exercise in patients on dialysis: a multicenter, randomized clinical trial. J Am Soc 
Nephrol. 2017;28(4):1259-68. The largest randomized clinical trial on home-based exercise in patients on dialysis, with an easily implementable unsupervised home exercise regimen.

43. Jhamb M, McNulty ML, Ingalsbe G, et al. Knowledge, barriers and facilitators of exercise in dialysis patients: a qualitative study of patients, staff and nephrologists. BMC Nephrol. 2016;17(1):192.

44. Painter PL, Hector L, Ray K, Lynes L, Dibble S, Paul SM, et al. A randomized trial of exercise training after renal transplantation. Transplantation. 2002;74(1):42-8.

45. Greenwood SA, Koufaki P, Mercer TH, Rush R, O'Connor E, Tuffnell R, et al. Aerobic or resistance training and pulse wave velocity in kidney transplant recipients: a 12-week pilot randomized controlled trial (the exercise in renal transplant [ExeRT] trial). Am J Kidney Dis. 2015;66(4):689-98.
46. Kobashigawa J, Dadhania D, Bhorade S, et al. On behalf of the AST KPCOP Frailty Workgroup. Report from the American Society of Transplantation on frailty in solid organ transplantation. Am J Transplant 2018; [Epub ahead of print]. The recent consensus document from the American Society of Transplantation's Kidney-Pancreas Community of Practice, with summary of the current state of frailty and directions for clinical practice and research.

47. Tullius SG, Tran H, Guleria I, Malek SK, Tilney NL, Milford E. The combination of donor and recipient age is critical in determining host immunoresponsiveness and renal transplant outcome. Ann Surg. 2010;252(4):662-74.

48. Lepeytre F, Dahhou M, Zhang X, Boucquemont J, Sapir-Pichhadze $\mathrm{R}$, Cardinal H, et al. Association of sex with risk of kidney graft failure differs by age. J Am Soc Nephrol. 2017;28(10):3014-23. 